\title{
Classical nodular sclerosis Hodgkin lymphoma presenting with atypical cardiac involvement
}

\author{
Georgiana Butura*,1, Ion Antohe ${ }^{2}$, Catalin Danaila ${ }^{2}$, Alexandru Gluvacov ${ }^{3}$, Dan \\ Ferariu $^{4}$, Mugurel Bosanceanu ${ }^{5}$, Angelica Slatineanu ${ }^{6}$, Amalia Merticariu ${ }^{3}$, Walther \\ Bild $^{7}$, Angela Dascalescu ${ }^{2}$
}

1"Gr. T. Popa" University of Medicine and Pharmacy, lasi, ${ }^{2}$ Hematology Department, "Gr. T. Popa" University of Medicine and Pharmacy, lasi, ${ }^{3}$ Hematology Department, Regional Oncology Institute, lasi, ${ }^{4}$ Department of Pathology, Regional Oncology Institute, lasi, ${ }^{5}$ Thoracic Surgery Department, Regional Oncology Institute, lasi, ${ }^{6}$ Radiology Department, Regional Oncology Institute, lasi, ${ }^{7}$ Physiology Department, "Gr. T. Popa" University of Medicine and Pharmacy, lasi, Romania

\begin{abstract}
Primary and secondary cardiac involvements in lymphomas are extremely rare and sometimes ill-defined entities. Thorough clinical and imagistic investigation is crucial to evaluate disease extension and its impact on cardiac function. Chemotherapeutic agents with potential cardiovascular toxicity must be cautiously employed in high risk patients. We present the case of a young female patient with classical limited stage Hodgkin lymphoma and unfavorable prognostic factors, cardiac tumor mass with significant mediastinal extension, pleural effusion and subsequent cardiac dysfunction. Potentially cardiotoxic ABVD regimen yielded complete remission and progressive reversal of parameters of cardiac function.
\end{abstract}

Keywords: Hodgkin lymphoma, cardiac tumor, Doxorubicin

\section{Introduction}

Primary cardiac tumors represent rare entities with an estimated necroptic incidence of $0.05 \%-1 \%$. Secondary cardiac involvement, regardless of the primary tumor histology, is more frequent (0.7-3.5\% incidence) [1-4].

Little attention has been directed towards cardiac involvement in lymphoma patients [5]. Primary cardiac lymphoma represents roughly $1.8 \%$ of cardiac tumors; nevertheless, secondary cardiac determinations in patients with disseminated disease are more frequent [2]. The incidence of cardiac determinations in

Received: December 2015; Accepted after review: February 2016; Published: March 2016.

*Corresponding author: Georgiana Butura, PhD Student, „Gr. T. Popa” University of Medicine and Pharmacy, lasi, Romania

Email: geodya@yahoo.com
Hodgkin lymphoma $(\mathrm{HL})$ remains unknown.

Clinical manifestations of cardiac tumors, irrespective of histological type, include palpitations, dyspnea, fatigue, chest pain and evidence of heart failure or pericardial effusion, reflecting the effect of the tumor mass on cardiac and pericardium anatomy and function. Common involved sites include the pericardium, atrial and ventricular walls, interventricular and interatrial septa. Tumor masses are usually polylobated and can present with either endocavitary expression and cardiac flow obstruction, or predominant pericardial involvement. Pericardial effusions are a common finding $[6,7]$.

Cardiac imaging techniques are of utmost importance in the diagnosis of cardiac tumors. Two-dimensional (2-D) transthoracic echocardiography (TTE) is commonly used due to its availability and general information provided concerning approximate tumor mass 
size, topography, overall impact on cardiac function and presence of pericardial effusion. Disadvantages of TTE include a potentially restricted field of view and underestimation of tumor extension. Cardiac computed tomography (CT), magnetic resonance imaging (MRI) and positron emission tomography (PET) are further required to fully characterize tumor size and density, myocardial infiltration and metabolic activity [4, 8, 9].

Careful screening for myocardial dysfunction in patients with primary or secondary cardiac lymphomas is of utter importance, since it can limit the use of several therapeutic agents, including anthracyclins, commonly used in lymphoma therapy.

\section{Case report}

We present the case of a previously healthy 34 year-old female patient admitted in the cardiology department for fatigue, significant weight loss during the previous 8 months, sweating, rest dyspnea, productive cough and anterior thoracic pain. The onset of the disease was 3 months before admission and the symptoms gradually worsened.
Clinical examination revealed left supraclavicular $1.5 \mathrm{~cm}$ lymphadenopathy, 2 $\mathrm{cm}$ below the costal margin hepatomegaly, a grade 3 mitral systolic murmur and grade 2 turgid jugular veins.

Echocardiography showed a 70/28 mm tumor mass originating in the interatrial groove, with left ventricular wall extension, minimal mitral regurgitation and a left ventricular ejection fraction (LVEF) of $50 \%$. No spontaneous contrast effect, indicative of possible thrombosis, was present. The patient presented also a circumferential pericardial effusion with $50 \mathrm{~mm}$ maximum posterior thickness.

A thoracic CT examination confirmed the presence of a 169/93/94 $\mathrm{mm}$ polylobated tumor mass originating in the visceral pericardium and infiltrating the subjacent myocardium. The tumor surrounded the emergence of the aorta, the aortic arch, left carotid and subclavian arteries and main pulmonary artery. A cranial expansion of the tumor engulfed the left carotid neurovascular bundle. Massive pericardial effusion, superficially situated from the tumor mass, was associated, as well as important inferior vena cava dilatation $(30 \mathrm{~mm})$ and mild hepatomegaly (Figure 1).

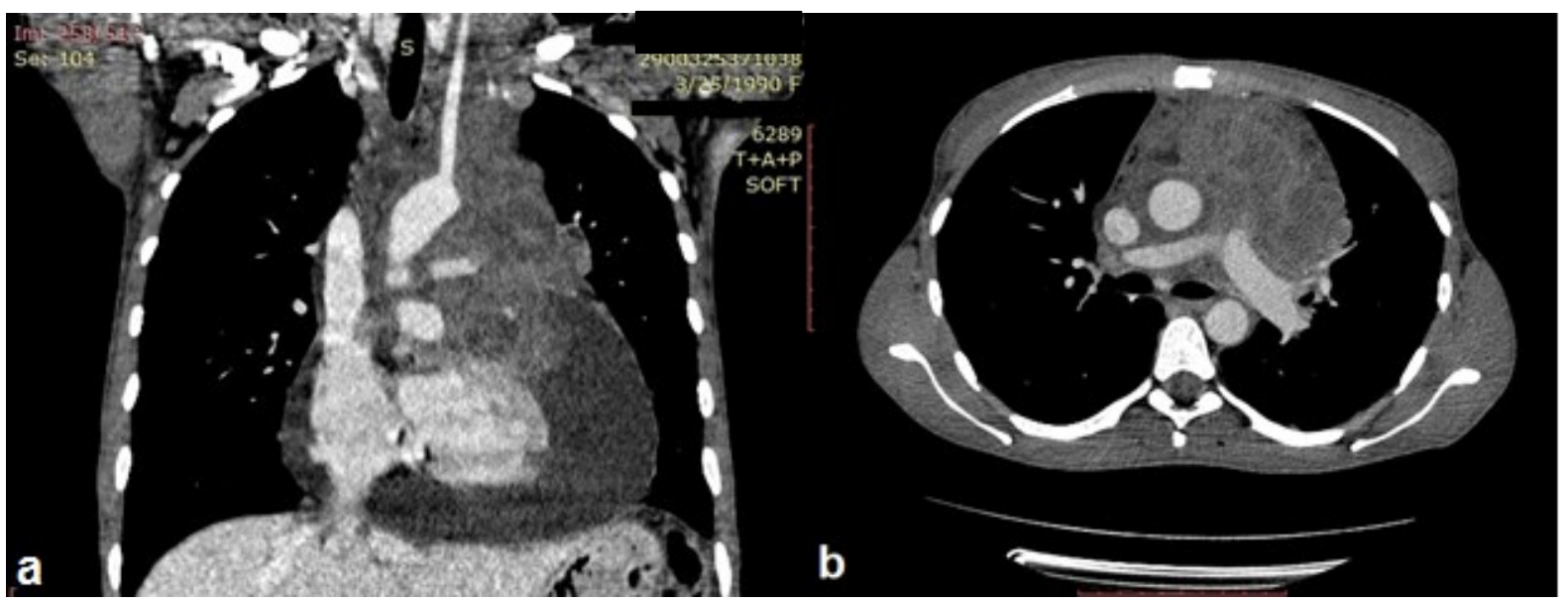

Fig. 1. Thoracic CT scan: a) coronal section and $\mathbf{b}$ ) axial section in a patient with polylobated tumor mass originating in the visceral pericardium and infiltrating the subjacent myocardium with massive pericardial effusion. The tumor surrounded the emergence of the aorta, the aortic arch, left carotid and subclavian arteries and the pulmonary artery. 
An emergency pericardiocentesis was performed and $2200 \mathrm{~mL}$ of pericardial fluid were evacuated during a 3 day period. Pleuropericardial window and pleural drainage were performed by video-assisted mini thoracotomy.
Pericardial biopsy revealed multiple nodules with interstitial hyalineosis containing a polymorphic infiltrate of small lymphocytes, rare eosinophils and Reed-Sternberg cells with CD30 and CD15 positivity (Figure 2).

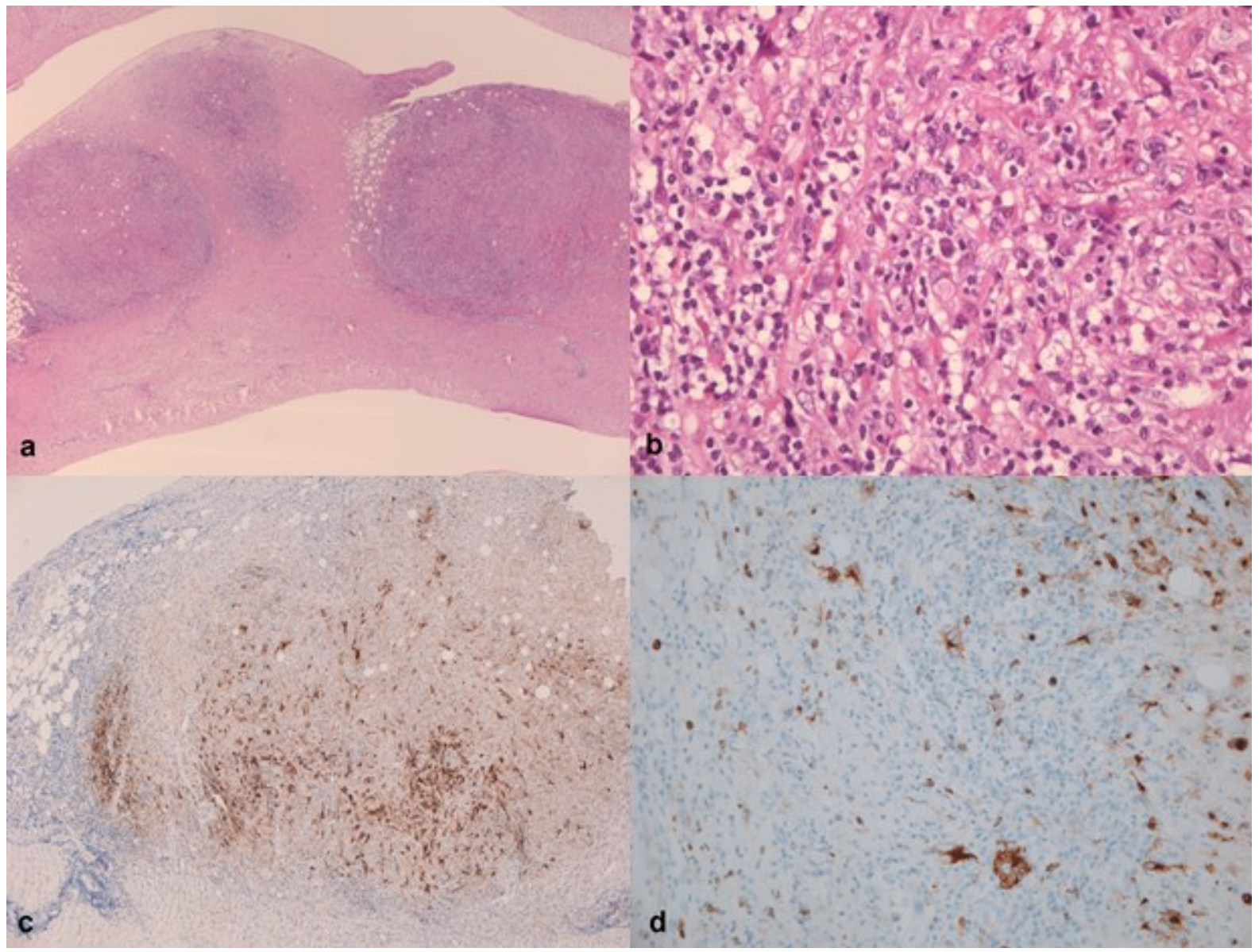

Fig. 2. Histopathological and immunohistochemical aspects: a) nodular pericardial infiltrates (HE, x25); b) ReedSternberg cells in a lymphocytic background (HE, x400); c) CD30 positive tumor cells (IHC, anti-CD30 Ab, x 50);

d) CD15, Golgi staining of Reed-Sternberg cells (IHC, anti-CD15 Ab, x200).

Biologic evaluation revealed mild anemia (hemoglobin $11.6 \mathrm{~g} / \mathrm{dL}$ ) and inflammatory syndrome. Serum creatine kinase MB and MM isoforms were normal, as well as troponin $\mathrm{T}$ levels. High $\mathrm{N}$-terminal prohormone of brain natriuretic peptide (NT Pro-BNP) serum levels of $2778 \mathrm{pg} / \mathrm{mL}$ suggested the severity of cardiac dysfunction [10]. A diagnosis of classical nodular sclerosis $\mathrm{HL}$ was thus established. No further tumor masses were discovered at abdominal and pelvic CT scan and bone marrow biopsy was normal, allowing us to define stage $\|_{E}$ at diagnosis [11]. Prognostic factors were evaluated according to the European Organization for the Research and Treatment of Cancer (EORTC) guidelines for early stage HL [12]. The patient presented 2 unfavorable features: 169/93/94 mm bulky tumor mass and elevated erythrocyte sedimentation rate $(E S R>50 \mathrm{~mm} / \mathrm{h}$ ).

Standard ABVD (Adriblastine $20 \mathrm{mg} / \mathrm{m}^{2}$, Bleomycin $10 \mathrm{mg} / \mathrm{m}^{2}$, Vinblastine $6 \mathrm{mg} / \mathrm{m}^{2}$, Dacarbazine $375 \mathrm{mg} / \mathrm{m}^{2}$ ) therapy was started and the patient received a total of 6 cycles, with no hematologic toxicity and immediate clinical improvement after first cycle.

Post therapeutic CT scan showed a restant mediastinal tumor mass $(>50 \%$ reduction in size) and disappearance of cardiac involvement (Figure 3). 


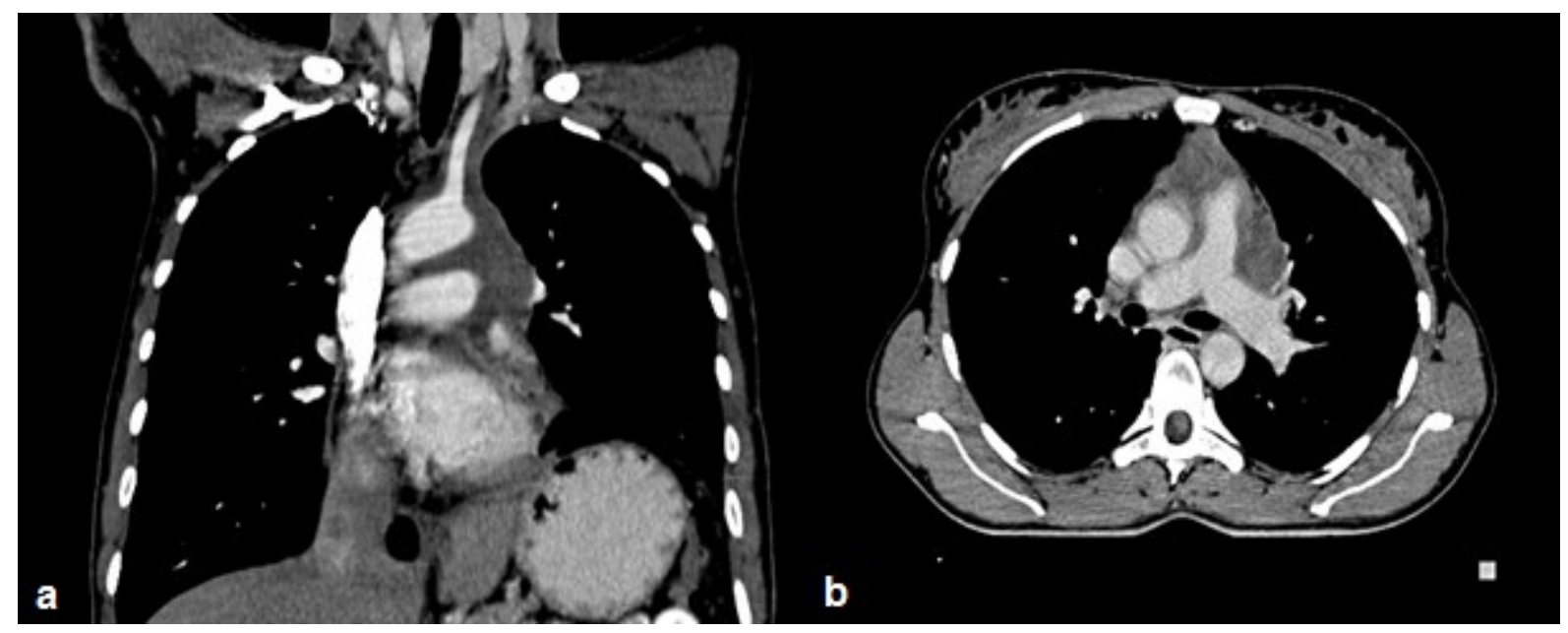

Fig. 3. Thoracic CT scan: a) coronal section and b) axial section in a patient with residual cervicomediastinal tumor mass. No pericardial effusion is seen. (Following chemotherapy completion).

PET scan revealed no metabolic activity of the residual tumor mass, confirming the complete therapeutic response (Figure 4).

TTE after chemotherapy completion revealed a normal LVEF of $70 \%$, free cavities, intact septa and no pericardial effusion.
Normal NT Pro BNP was noted at this moment.

The patient is currently in complete remission and is being reevaluated every 6 months according to our institutional protocol.

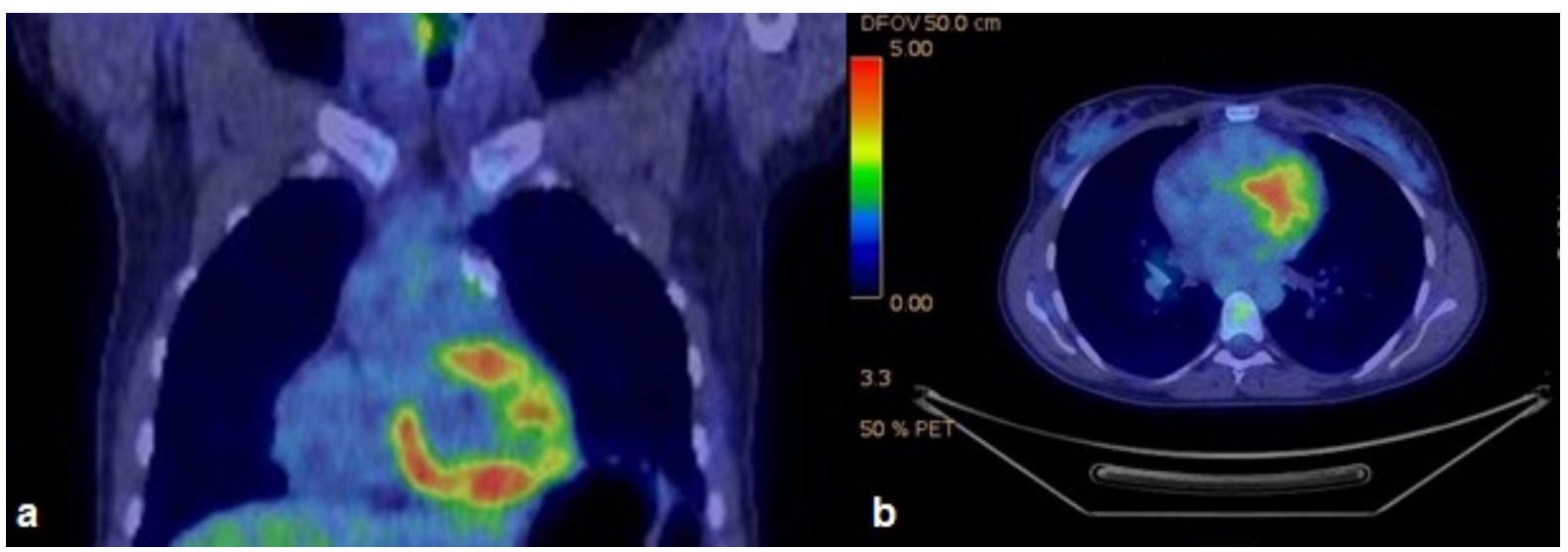

Fig. 4. Thoracic PET-CT scan: a) coronal section and b) axial section showing metabolic inactivity in residual mediastinal masses.

\section{Discussion}

Lymphomas account for $55.6 \%$ of hematological malignancies and $\mathrm{HL}$ represents $30 \%$ of total lymphoma cases [13]. Pulmonary, hepatic, cerebral and testicular tissues are among the most common extranodal determinations.
Cardiac involvement is rarely described in the literature. Amirimoghaddam et al. describes an isolated pediatric case of advanced stage $\mathrm{HL}$ with cardiac involvement in which tumour extension involved the entire heart tissue, liver, spleen and bone marrow [5]. The patient was treated with ABVD regimen and achieved complete remission. Lymphomas without extranodal determinations 
are generally associated with chemosensitivity and better outcome. The presence of extraganglionar involvement has been linked to poorer prognosis, elevated serum lactate dehydrogenase $(\mathrm{LDH})$ levels, advanced stage disease and central nervous system infiltration $[12,14]$.

Cardiac lymphomas have a greater incidence in Epstein Barr positive and immunocompromised individuals (acquired immunodeficiency syndrome patients, solid organ transplant recipients) [15]. Cardiac involvement can be either primary (tumor mass limited to myocardium and pericardium) or secondary [16]. Dissemination can occur by lymphatic, bloodstream or contiguous extension [2, 17]. Imagistic examination (ultrasonography, CT, PET/CT) has a significant role in establishing the degree of myocardial and pericardic infiltration, extracardiac dissemination and heart function [8].

$\mathrm{HL}$ staging is performed according to the Ann Arbor classification, allowing practitioners to distinguish between early stage (stages I and II) and advanced stage (stages III and IV) disease. The patient risk is further stratified by evaluating specific risk factors for limited and advanced disease [11]. Risk factors for early stage $\mathrm{HL}$ include bulky disease, involvement of more than 3 nodal sites, extranodal involvement and elevated ESR [12]. This classification has impact on treatment selection and must be thoroughly performed in HL patients [15].

Standard therapeutic guidelines in $\mathrm{HL}$ are promoting the anthracyclin - containing regimen $A B V D$ as front line therapy in patients with stage II disease and unfavourable prognostic factors [18]. Doxorubicin is an anthracyclin antibiotic whose antineoplastic activity is based on its topoisomerase II inhibitory function. It is a highly cardiotoxic compound and may determine acute or chronic cardiac toxicity, should a total dose of $550 \mathrm{mg} / \mathrm{m}^{2}$ be surpassed. Close patient monitoring is essential to decrease the incidence of anthracycline - induced cardiotoxicity, as is the early implementation of cardioprotective therapies in high risk individuals [19].
The most promising solution for preventing cardiotoxicity is the coadministration of dexrazoxane, an iron chelating agent that reduces the formation of anthracycline-iron complexes [20, 21]. It has been demonstrated that high dose doxorubicin in patients with preexistent cardiac dysfunction (evidenced by increased troponin I levels, a 10\% LVEF decrease below the normal limit, $20 \%$ at any level or an absolute LVEF of 45\%) is associated with further functional cardiac deterioration in the following 7 months [19, 22].

We presented the case of a young female patient with early stage classical $\mathrm{HL}$ and 2 unfavorable prognostic factors. Association of extranodal cardiac involvement with pericardial effusion and significant impairment of cardiac function, as indicated by LFEF and NT Pro BNP serum levels, is an aditional unfavourable feature, potentially limiting the use of cardiotoxic anthracyclines. Evaluation of patient eligibility for cardiotoxic therapy in cardiac lymphomas is not standardised and must be carefully weighed in each clinical setting. It should be noted that transthoracic ultrasonography can sometimes underestimate the actual quantity of pericardial fluid, underlining the utter importance of cardiac CT and IRM examination. Emergency pericardiocenthesis was indicated in this case, leading to immediate improvement of cardiac function.

The differentiation between a primary cardiac tumour with cervico mediastinal extension and a mediastinal tumour infiltrating the myocardium is difficult.

In this case, myocardial tumoral infiltration extending from visceral pericardium supports the first hypothesis. However, post therapy persistence of mediastinal tumor and disappearance of all heart masses could stand for mediastinal lymphoma with secondary cardiac infiltration. It should be noted that an actual unanimously accepted definition of primary cardiac lymphoma, allowing practitioners to separate this rare entitity from the more frequent mediastinal lymphomas with secondary cardiac involvement, does not exist.

In the absence of hepatic biopsy it is difficult to ascertain whether hepatomegaly represents the expression of secondary 
lymphoma determination or the indirect result of retrograde vena cava pressure increase.

We opted for ABVD treatment after considering the bulky tumor mass, patient age and potentially favourable therapeutic following standard therapy. Doxorubicin was administred during intensive 24 hours surveillance in the intensive care unit.

Cardioprotective therapy in the form of dexrazoxane was not available. Nonetheless, no acute cardiac adverse events were noted and cardiac function was markedly improved after chemotherapy. Long term monitoring of cardiac function is required in such cases.

\section{References}

1. Amano J, Nakayama J, Yoshimura Y, Ikeda U. Clinical classification of cardiovascular tumors and tumor-likelesions, and its incidences. Gen Thorac Cardiovasc Surg 2013; 61:435-447.

2. Butany J, Nair V, Naseemuddin A, Nair GM, Catton C, Yau T. Cardiac tumours: diagnosis and management. Lancet Oncol 2005; 6:219228.

3. Maraj S, Pressman GS, Figueredo VM et al. Review Primary Cardiac Tumors. Int $J$ Cardiol 2009; 133:152-156.

4. Miguel CE, Bestetti RB. Primary cardiac lymphoma. Int J Cardiol 2011; 149:358-363.

5. Amirimoghaddam Z, Khoddami M, Nayeri ND, Molaee S. Hodgkin's lymphoma presenting with heart failure: a case report. J Med Case Rep 2010; 4:14.

6. Rolla G, Calligaris-Cappio F, Burke AP. Cardiac lymphomas. In Travis WD, Brambilla E, Müller-Hermelink HK, Harris CC (eds.) World Health Organization. Pathology and genetics of tumours of the lung, pleura, thymus and heart. Lyon: IARC Press; 2004:282-286.

7. Petrich A, Cho SI, Billett $\mathrm{H}$. Primary cardiac lymphoma: an analysis of presentation, treatment, and outcome patterns. Cancer 2011; 17(3):581-589.

8. Habertheuer A, Ehrlich M, Wiedemann D, Mora B, Rath C, Kocher A. A rare case of primary cardiac B cell lymphoma. J Cardiothorac Surg 2014; 9:14.

9. Leja MJ, Shah DJ, Reardon MJ. Primary Cardiac Tumors. Tex Heart Inst J 2011; 38(3):261-262.

10. Expert Group on Biomarkers. Biomarkers in Cardiology - Part 1 - In Heart Failure and Specific Cardiomyopathies. Arq Bras Cardiol 2014; 103(6):451-459.

\section{Conclusion}

Cardiac involvement in lymphomas is a rare occurrence. We presented a case of classical $\mathrm{HL}$ in a young female patient with cardiac determination and bulky mediastinal tumor mass and unfavorable prognostic features. Despite the high risk of anthracyclinrelated cardiac toxicity no cardiovascular adverse events were noted and post therapeutic imagistic evaluation revealed complete remission and reversal of cardiac dysfunction.
11. Lister TA, Crowther D, Sutcliffe SB, et al. Report of a committee convened to discuss the evaluation and staging of patients with Hodgkin's disease: Cotswolds meeting. J Clin Oncol 1989; 7(11):1630-1636.

12. Engert $A$, Eichenauer DA, Dreyling M; ESMO Guidelines Working Group. Hodgkin's lymphoma: ESMO Clinical Practice Guidelines for diagnosis, treatment and follow-up Ann Oncol 2010; 21(5):168-171.

13. Jaffe ES. The $2008 \mathrm{WHO}$ classification of lymphomas: implications for clinical practice and translational research. Hematology Am Soc Hematol Educ Program 2009; 523-531.

14. National Comprehensive Cancer Network. Hodgkin's Lymphoma in NCCN Clinical Practice Guidelines in Oncology. V.2.2015.

15. Holladay AO, Siegel RJ, Schwartz DA. Cardiac malignantlymphoma in acquired immune deficiency syndrome. Cancer 1992; 70:22032207.

16. Montalbetti L, Della Volpe A, Airaghi ML, Landoni C, Brambilla-Pisoni G, Pozzi S. Primary cardiac lymphoma.A case report and review. Minerva Cardioangiol 1999; 47:175182.

17. Timóteo AT, Gouveia R, Gonçalves PA et al. Lymphoma with Clinical Presentation of a Primary Pericardial Tumor. Rev Port Cardiol 2003; 22(11):1385-1391.

18. Eich HT, Diehl V, Görgen $H$, et al. Intensified chemotherapy and dose-reduced involved-field radiotherapy in patients with early unfavorable Hodgkin's lymphoma: final analysis of the German Hodgkin Study Group HD11 trial. J ClinOncol 2010; 28(27):4199-4206. 
19. Volkova M, Russell R. Anthracycline Cardiotoxicity: Prevalence, Pathogenesis and Treatment. Curr Cardiol Rev 2011; 7:214-220.

20. Zuppinger C, Timolati F, Suter TM. Pathophysiology and diagnosis of cancer drug induced cardiomyopathy. Cardiovasc Toxicol 2007; 7:61-66.
21. Hochster HS. Clinical pharmacology of dexrazoxane. Semin Oncol 1998; 25(10):37-42.

22. Cardinale D, Sandri MT, Martinoni A. Myocardial injury revealed by plasma troponin I in breast cancer treated with high-dose chemotherapy. Ann Oncol 2002; 13(5):710715 . 